Supporting information for

\title{
Elucidating the Contributions of Plasmon-Induced Excitons and Hot Carriers to the Photocurrent of Molecular Junctions
}

\author{
Yoram Selzer* \\ Department of Chemical Physics \\ School of Chemistry, Tel Aviv University, Tel Aviv 69978, Israel
}

\section{Experimental section}

Synthesis of tetrathiafulvalene derivative: 4,4'(5')-Diformyltetrathiafulvalene (DF-TTF) was synthesized following a published procedure ${ }^{\mathrm{S} 1}$.

Growth and characterization of the TTFW monolayer: The lithography defined $\mathrm{Au}$ leads were immersed in $1 \mathrm{mM}$ 4-aminothiophenol solutions in argon-purged absolute ethanol for overnight assembly (step I). After thorough rinsing the sample was immersed in a $10 \mathrm{mM}$ DF-TTF solution in DMSO with $5 \mu \mathrm{L}$ of $\mathrm{HCl}(37 \%)$ as an acid catalyst for $24 \mathrm{~h}$ (step II). After thorough rinsing the sample was immersed in a $10 \mathrm{mM}$ DMSO solution of Aniline, for the final growth step (step III). The growth process was monitored by Reflection-Absorption Infrared Spectroscopy of a large Au pad defined on the same $\mathrm{SiO}_{2} / \mathrm{Si}$ substrate used for all the contact leads for the junctions. Step I was determined by the presence of the amine peak at $3360 \mathrm{~cm}^{-1}$ (figure S1). In step II the amine peak disappears and the CO stretch peak of DF-TTF appears at $1670 \mathrm{~cm}^{-1}$. Step III is monitored by the disappearance of this peak. 
III

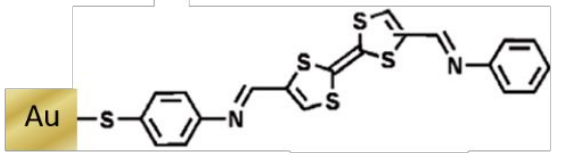

II
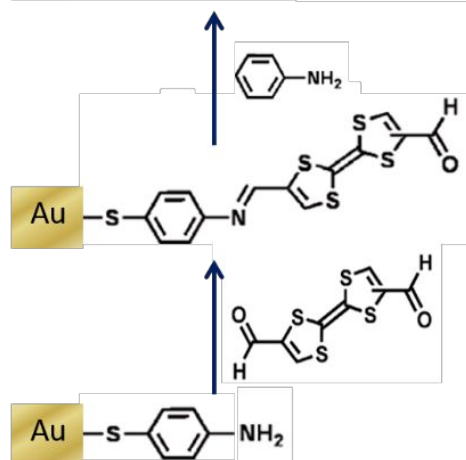

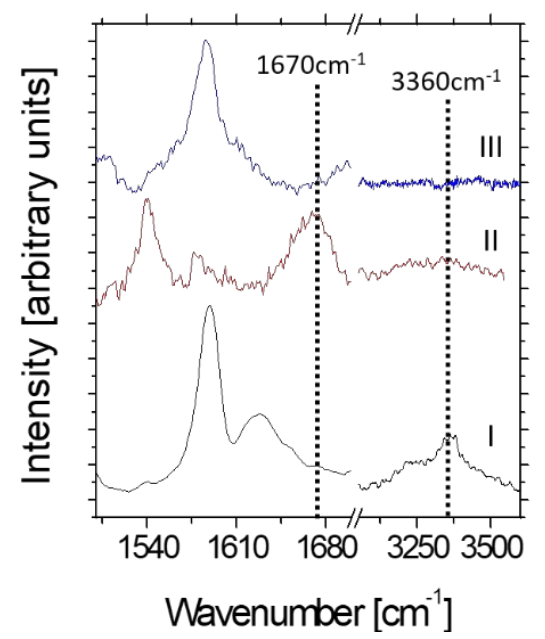

Figure S1. Growth scheme of the TTFW monolayer and the characterization of this process by IR.

Optical properties of the TTFW monolayer: UV-Vis spectra of glass slides covered with $10 \mathrm{~nm}$ of Au and a TTFW monolayer were measured to produce the black spectrum in figure 2 .

Calculation of the plasmon resonance of SWMJ: The blue curve in figure 2 was calculated by a commercial FDTD software (Lumerical) using the built-in tabulated dielectric values of $\mathrm{Au}$ for the two wavelengths. The geometry of the calculated structure is depicted in figure S2. The size of the molecular gap in this figure is $10 \mathrm{~nm}$, only for purposes of visibility, in order to better show graphically the enhanced field within the junction. In the calculation used for figure 2 in the main text, the gap size was $2.5 \mathrm{~nm}$. All the other structural parameters are the same. 


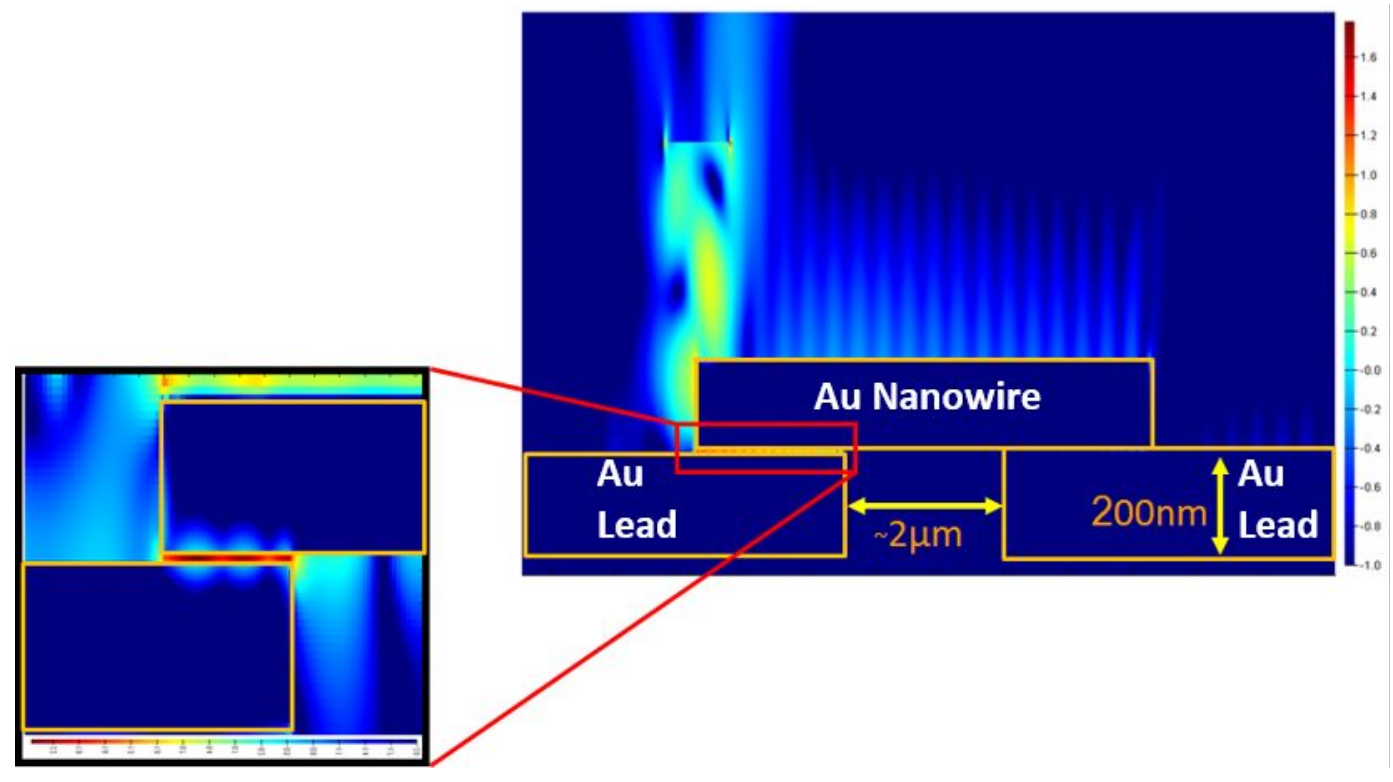

Figure S2. The SWMJ structure used for the FDTD simulation of the plasmon resonance.

Transition Voltage Spectroscopy: The $V_{T}$ value of OPE junctions has previously been determined to be $0.80 \pm 0.13 \mathrm{~V}$, in agreement with our measurements ${ }^{\mathrm{S} 2}$. TVS has never been performed for TTFW junctions. However, the structure of this molecule is similar to OPV(3) in reference $\mathrm{S} 2$, for which $V_{T}=0.57 \pm 0.07 \mathrm{~V}$, in par with our value.

It is important to emphasize the use of TVS in this study: The reproducibility of the $V_{T}$ values indicates junctions with the same potential divider across the two possible junctions that can be formed in each SWMJ structure. The fact that for OPE we measure similar $V_{T}$ values to those reported for individual junctions with the same molecule, support the argument that there is only one junction in each SWMJ. This point has been thoroughly discussed in the past, see references 29 and 39-41 in the main text. Following this reasoning we regard the similar spread of $V_{T}$ values measured for the TTFW junctions as an indication for the structural similarity and reproducibility in the sense that only one junction per structure can produce such a small spread of transition voltage values. The average value, as indicated above, has been measured using other types of junctions with molecules with a similar structure. 


\section{References}

S1. Andreu, R.; Garin, J.; Orduna, J. Tetrahedron Lett. 1994, 35, 9243-9246.

S2. Beebe, J. M.; Kim, B.; Frisbie, C. D.; Kushmerick, J. G. ACS Nano 2008, 2, 827832 . 